\title{
Recent highlights from SUSY searches at CMS
}

\author{
Denis Rathjens* ${ }^{\dagger}$ \\ University of Hamburg, Germany \\ for the CMS collaboration \\ E-mail: Denis.Rathjens@eern.ch
}

In these proceedings, the latest results of the CMS collaboration on searches for supersymmetric particle (sparticle) production at the LHC are reviewed. A variety of complementary final state signatures and methods are presented using $20 \mathrm{fb}^{-1}$ of data from the $\sqrt{s}=8 \mathrm{TeV}$ LHC run.

The variety of searches shown ranges from an inclusive all-jet search for strong production of sparticles over a search employing $\tilde{t}$-tagging techniques and a search for a di-leptonic mass edge, jets and $\not_{\mathrm{T}}$ to the first search for vector boson fusion (VBF) production of $\tilde{\tau}$ in a compressed mass-spectrum scenario. In summary, the large coverage of supersymmetric parameter space by the CMS collaboration is demonstrated.

XXIII International Workshop on Deep-Inelastic Scattering,

27 April - May 12015

Dallas, Texas

* Speaker.

$\dagger$ I would like to thank the LHC colleagues for the outstanding performance of the accelerator, and the many members of the CMS collaboration who, from detector operation to analysis, made it possible for so many state-of-the art results to be presented at this conference. 


\section{Introduction}

The Standard Model of particle physics (SM) can be considered to be a low energy effective theory. In such a case, the radiative corrections to the Higgs-boson mass would diverge quadratically. Since fermions and bosons have opposite sign contributions in radiative correction loops to masses, a good solution to such a problem could be to introduce partner particles to each known particle that have a half-integer difference in spin. Such an extension to the SM is denoted as Supersymmetry (SUSY) and would have many more attractive consequences like a possible unification of forces at high energies or the introduction of a dark matter candidate in the case of R-parity conservation.

The downside of SUSY is that sparticles with the same mass as SM particles would have been found, already. Therefore, SUSY needs to be a broken symmetry and as such allows for a variety of parameters and breaking mechanisms that span a huge parameter space challenging to cover.

In these conference proceedings, excerpts of selected recent research results of the CMS collaboration are presented that cover large parts of the minimal supersymmetric model (MSSM) parameter space complementarily.

More specifically, strong squark- or gluino- mediated production of squarks searched for via the $\mathrm{M}_{\mathrm{T} 2}$ variable quantifying the hadronic recoil for an inclusive search [1], production of heavy stop quarks leading to boosted top decays that are resolved with a boosted top-tagging algorithm [2], sbottom production with leptonic decays leading to a mass-edge in the di-leptonic mass-spectrum in association with jets and missing transverse energy [3], and the first search of its kind for compressed mass spectrum SUSY in vector boson fusion (VBF) production of staus [4] are reported. Only excerpts of the aforementioned analyses as published at the time of the conference are shown. The full and complete list of SUSY results from the CMS collabration can be found at [5].

\section{Inclusive all-jet search with hadronic recoil}

An inclusive search for supersymmetry in final states with only jets was pursued in CMS utilizing $H_{T}, \not_{\mathrm{T}}$, and $H_{T} \times \not_{\mathrm{T}}$ triggers. A veto on isolated leptons was required. Furthermore, a binning in the number of jets and b-tagged jets per event was undertaken in order to separate the different background contributions for the specified search regions. In total, nine regions as a function of the number of jets and b-quark tags times three different $H_{T}$ and $\phi_{\mathrm{T}}$ regions were defined.

The two decay channels discussed in these proceedings are gluino and squark production as shown in Fig. 1. In order to increase sensitivity, the hadronic event recoil was parametrized by a variable denoted as " $\mathrm{M}_{T 2}$ " based on a transverse mass definition as shown in Eq. 2.1.

$$
\left(M_{\mathrm{T}}^{(i)}\right)^{2}=\left(m^{\mathrm{vis}(i)}\right)^{2}+m_{\chi(i)}^{2}+2\left(E_{\mathrm{T}}^{\mathrm{vis}(i)} E_{\mathrm{T}}^{\chi(i)}-\vec{p}_{\mathrm{T}}^{\mathrm{vis}(i)} \cdot \vec{p}_{\mathrm{T}}^{\chi(i)}\right)
$$

Such a definition covers the case of a single invisible particle in a two body-decay showing an edge at the mother particle mass. For $R$ parity conserving SUSY, two invisible particles would leave the detector. Therefore, an extension to the classical transverse mass definition is undertaken that 

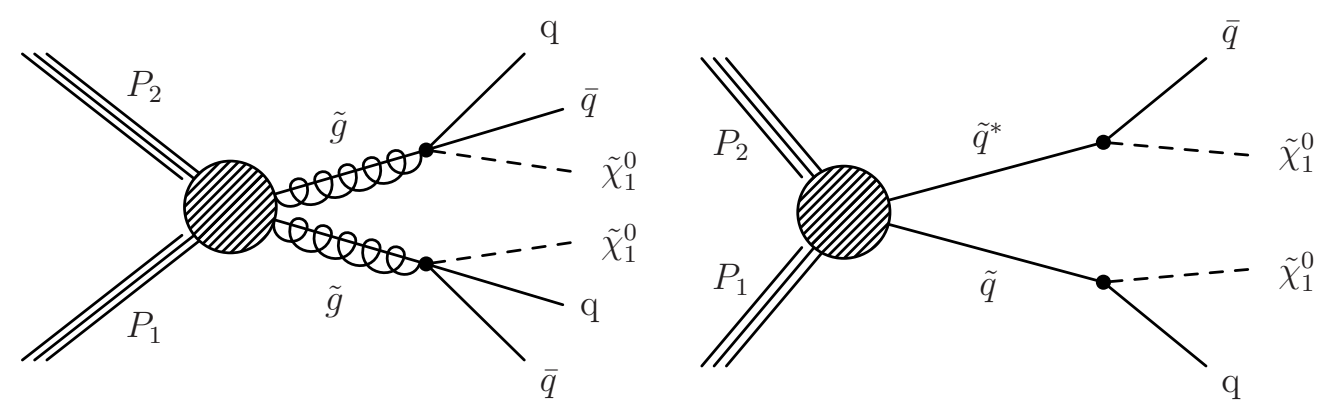

Figure 1: Simplified model scheme gluino (left) and squark (right) production.

builds an event hypothesis in the case of two LSPs leaving the detector, as shown in Eq. 2.2.

$$
M_{\mathrm{T} 2}\left(m_{\chi}\right)=\vec{p}_{\mathrm{T}}^{\chi(1)}+\vec{p}_{\mathrm{T}}^{\chi(2)}=\vec{p}_{\mathrm{T}}^{\operatorname{miss}}\left[\max \left(M_{\mathrm{T}}^{(1)}, M_{\mathrm{T}}^{(2)}\right)\right]
$$

High values of $\mathrm{M}_{T 2}$ could indicate the presence of two LSPs in an event. The major SM backgrounds of this method are constituted by tails of the jet energy resolution and leptons that have not been reconstructed. The background estimations for each search region were undertaken in a data-driven way. For multijet backgrounds, the requirement on the minimal separation in azimutal angle, $\phi$, was varied between the event recoil and the four leading jets. For leptonic $W+j e t$ and top production, single lepton sidebands were used. Estimating the $\mathrm{Z} \rightarrow v \bar{v}$ was done by utilizing photon or $\mathrm{Z} \rightarrow l \bar{l}$ events by subtracting the reconstructed lepton momenta from the event hypothesis. As no excess beyond the SM expectations was observed, limits have been set on various SUSY scenarios. As an example, the limits on gluino and light squark production are shown in Fig. 2.
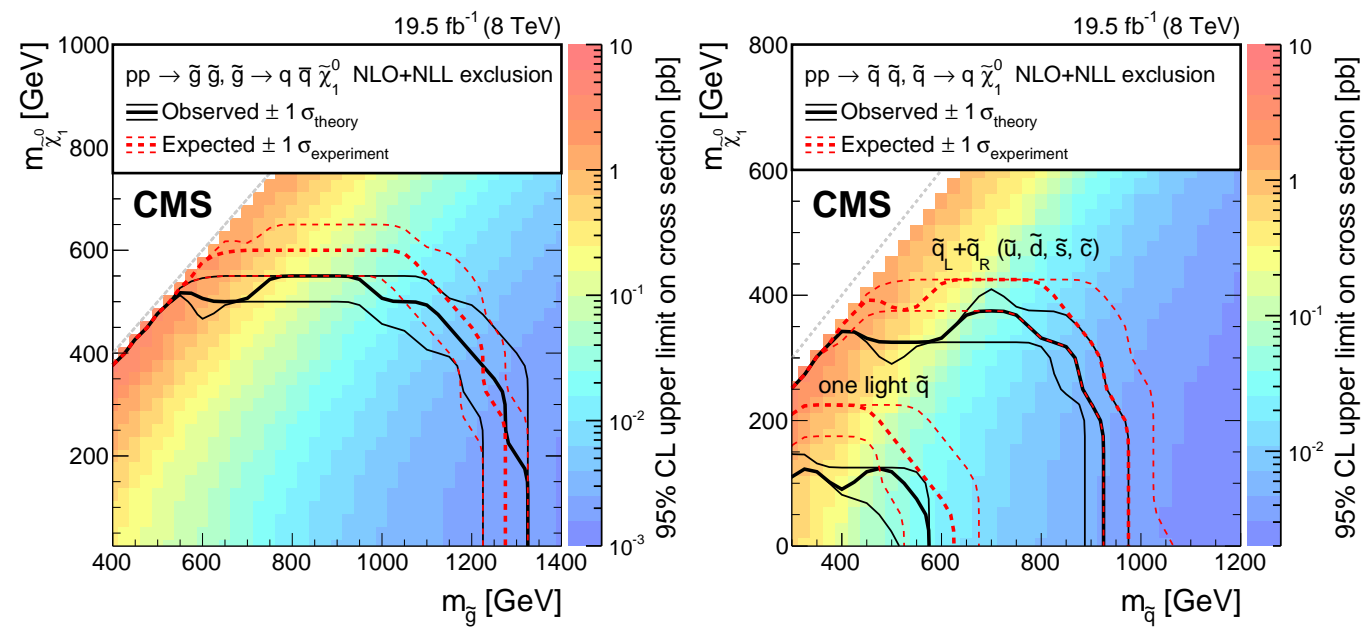

Figure 2: $95 \%$ confidence level (CL) exclusion limits for gluino production (left) and squark production (right). The latter plot shows two scenarios. One for the case of all light squark flavors being accessible that excludes a large part of the parameter space for the squark mass, the other for only one light squark of one handedness being accessible with a reduced exclusion power. 


\section{Hadronic search with $\tilde{t}$ tagging}

When searching for stop production, three rough cases have to be distinguished depending on the difference of the stop mass and LSP mass. Firstly, if the difference exceeds the W mass but not the top mass, the stop will dominantly decay via Wino to the LSP as shown in Fig. 3 (right). The visible decay products are the same as SM top decay products, but the kinematic properties are different. Secondly, if the difference exceeds the top mass slightly, a direct top plus LSP decay as shown in Fig. 3 (left) is dominant and the properties of the top quarks are those of SM top production. The only distinguishing quality lies within higher missing transverse momentum.

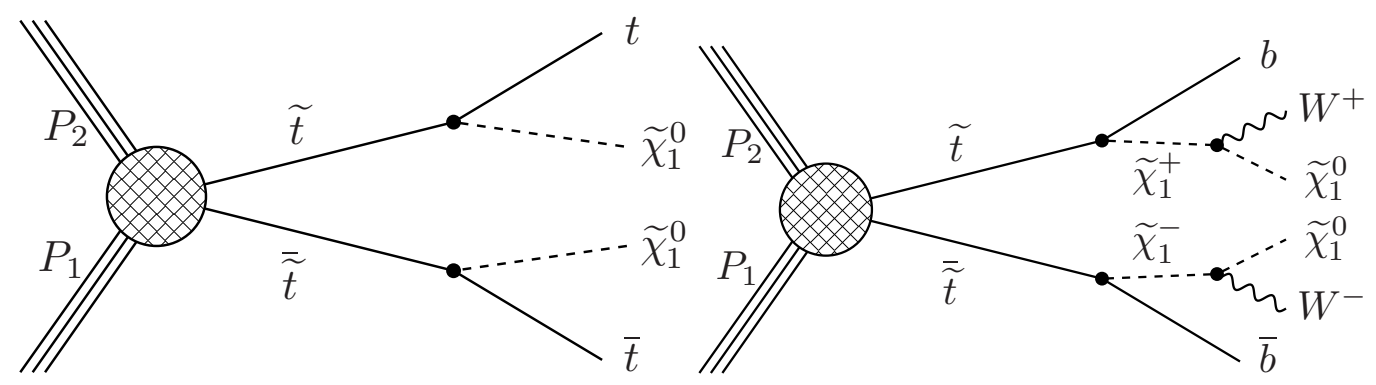

Figure 3: Simplified model scheme stop production decaying to either $t \tilde{\chi}_{1}^{0}$ (left) or $b \tilde{\chi}_{1}^{+}$(right).

Thirdly, if the difference exceeds the top mass by far, the top-decay products become boosted causing the reconstruction of several objects as one single jet. For such purposes, boosted toptagging techniques were employed that are based on the disassembly of jets with large jet radii and subsequent identification of merged objects. In combination with requiring a minimum separation of the angular distance in $\phi$ between any of the leading three jets and the missing transverse momentum, the remaining background of this search consists of SM top quark production. Two $\mathrm{M}_{\mathrm{T} 2}$-like variables $\left(\mathrm{M}_{\mathrm{T}}^{3 \text {-jet }}\right.$ and $\mathrm{M}_{\mathrm{T}}^{\mathrm{R} \text {-sys }}$ ) were used to gain sensitivity as shown in Fig. 4.
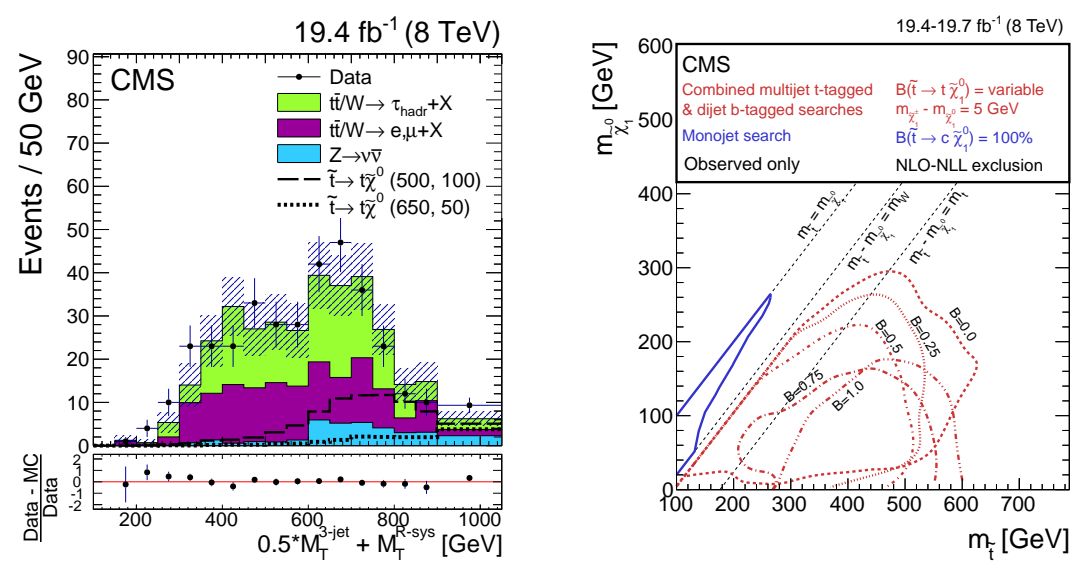

Figure 4: Multijet t-tagged signal region (left) and overall exclusion limits (right). A branching fraction (B) of 1.0 implies all decays are $\tilde{t} \rightarrow t \tilde{\chi}_{1}^{0} . \mathrm{B}=0.0$ implies all decays proceed throughout $\tilde{t} \rightarrow b \tilde{\chi}_{1}^{ \pm}$.

As no excess beyond the SM expectation was observed, limits have been set. In these proceedings, only the multijet t-tagged search is presented while the exclusion limits shown in Fig. 4 
(right) present the combination with a second search optimized for b-quark plus wino production.

\section{Search with a di-lepton mass edge, jets and $\not_{\mathrm{T}}$}

Searching for electroweak decay modes may allow for utilizing mass-edge techniques. In this search, e.g., a slepton mass edge (left) for slepton decays and a fixed edge (right) for zino decays have been sought as shown in Fig. 5. Dedicated di-lepton triggers were utilized and a mass
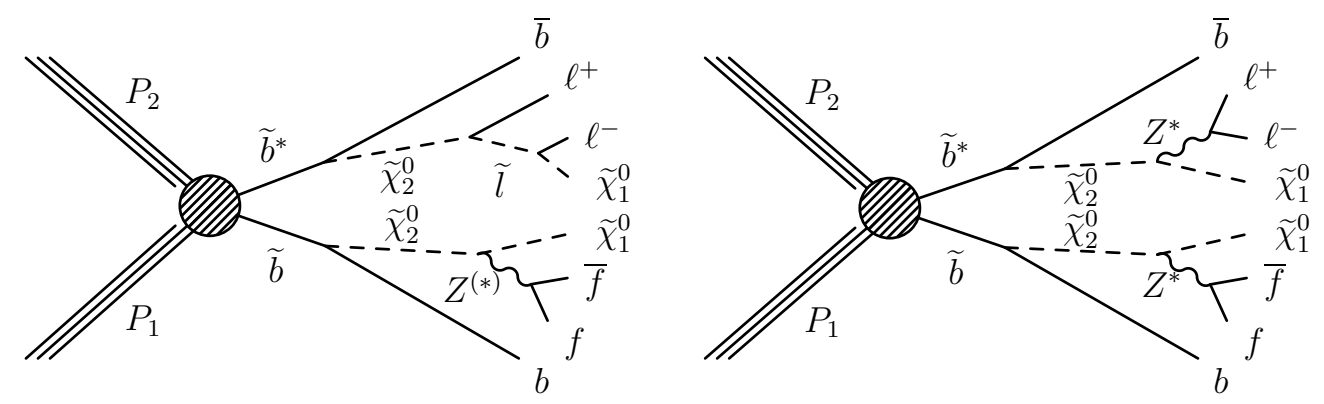

Figure 5: Simplified model scheme bino production decaying via slepton with slepton mass edge (left) or via zino with a fixed mass edge (right).

degeneracy of $\tilde{e}$ and $\tilde{\mu}$ was assumed. The main backgrounds are due to Drell-Yan production inside a Z-mass window or due to di-leptonic $t \bar{t}$ production. For the estimation of the $t \bar{t}$ background, it's flavor symmetric behavior was utilized. The contribution was measured in a sideband in a data. A fit of flavor symmetric, Drell-Yan and possible signal contributions was undertaken in regions below, on or above the Z-mass for two different detector regions each, under central tracker or endcap tracker coverage, respectively.

No evidence for a statistically significant signal was observed with a maximum deviation from the null hypothesis at the level of $2.6 \sigma$ within $20<m_{\overline{l l}}<70 \mathrm{GeV}$. Therefore, exclusion limits have been set as shown in Fig. 6 depending on the fixed (left) or slepton edge (right) scenario.
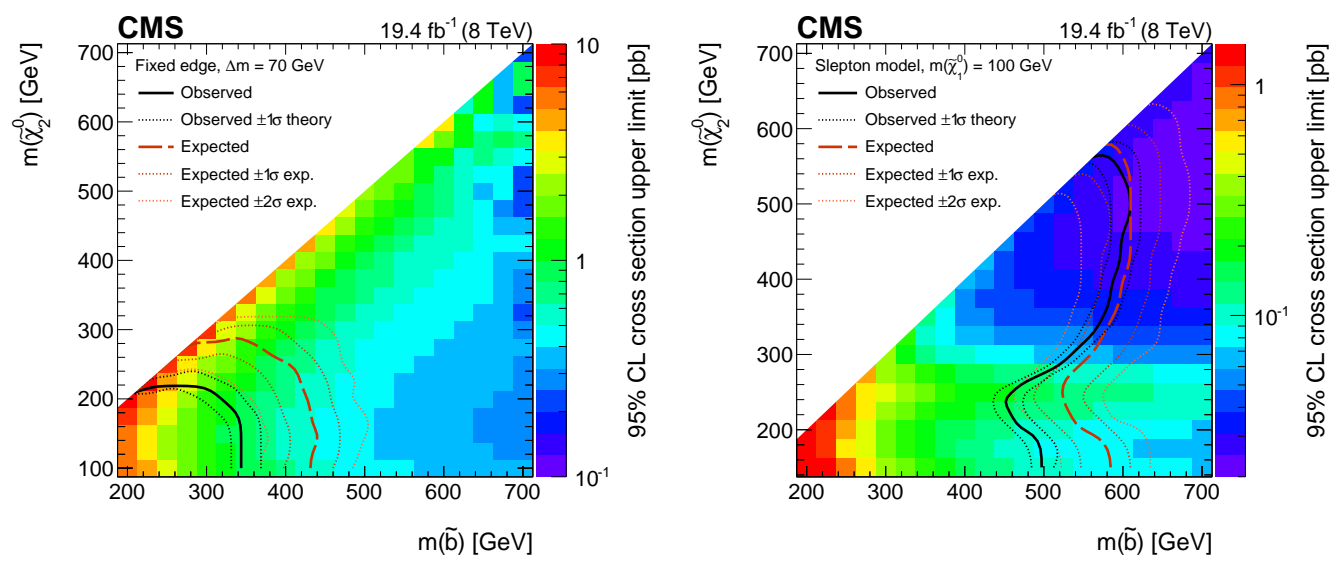

Figure 6: Exclusion limits for the fixed edge (left) and slepton edge (right) scenarios are presented in an interpretation for initial sbottom squark production. 


\section{Search for compressed spectrum SUSY in vector boson fusion $\tilde{\tau}$ production}

Finally, a search for compressed spectrum stau production was undertaken searching explicitly for vector boson fusion (VBF) production as shown in Fig. 7.

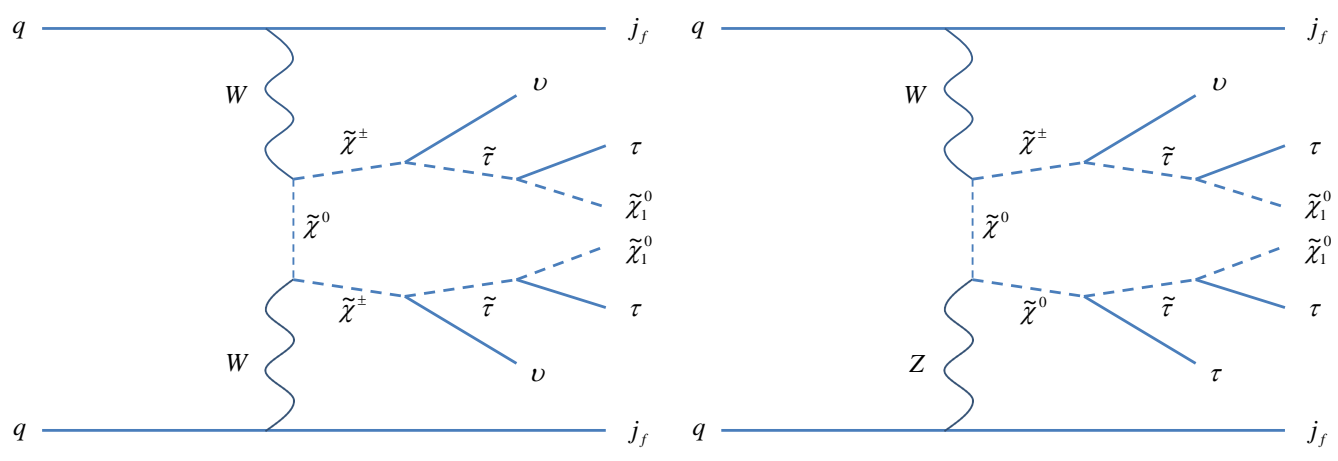

Figure 7: Vector boson fusion production of two (left) or three (right) $\tilde{\tau}$.

While VBF production itself is suppressed and cannot directly compete with direct electroweak production in terms of cross section, the t-channel exchange of vector bosons gives rise to a discriminative VBF-jet signature. The two initial state quarks form jets that are distinguished by a large separation in pseudorapidity, pointing to opposite hemispheres of the detector and relatively hard transverse momenta. While the decay channels and charges of the central $\tau \tau$ produced in the decay chains to the LSP determine the dominant backgrounds and the used triggers, the VBF jet tagging can always be used for background discrimination. Even standard model signatures with similar topologies like VBF Higgs production are suppressed, because they constitute s-channel production with different kinematic properties.

One of the main challenges of this analysis is the contribution of higher order SM processes to the backgrounds. $\mathrm{Z}+2$ jets is a background for all opposite sign channels, $\mathrm{W}+3$ jets is the main background for all channels containing a single hadronically decaying $\tau$, and 4 -jet multijet background is the main contribution to the channels with two hadronically decaying $\tau$. Therefore, most backgrounds were estimated in a data-driven way by inverting the VBF tag requirements, lepton isolation, vetoes on b-quarks, requiring the presence of three reconstructed leptons or a transverse mass window.

The best sensitivity is contributed from same-sign channels with low transverse momentum requirements on the trigger level. As such, the same sign di- $\mu$ channel as shown in Fig. 8 (left) is the most potent. Since no excess beyond SM expectations has been observed, limits (see Fig. 8 right) have been set. 

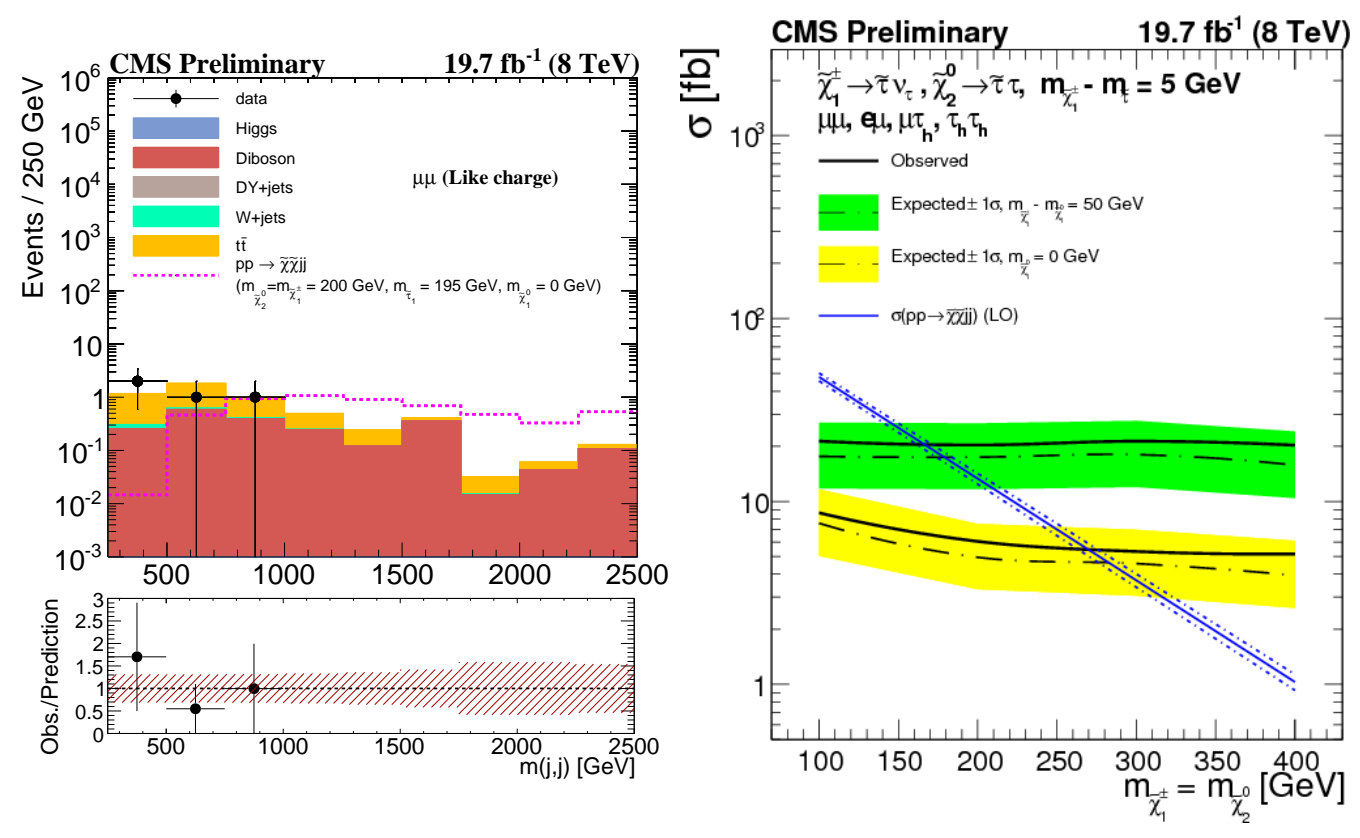

Figure 8: The most significant contribution to the exclusion limits (right) originates from the di- $\mu$ channel out of the decaying $\tau$ (left). Limits were set on two scenarios with an assumption of $100 \%$ branching ratio of charginos and neutralinos to $\tilde{\tau}$. The green case represents a low mass-difference between chargino or neutralino and the LSP (compressed spectrum). In the yellow case, the LSP was assumed to be massless.

\section{Summary}

The CMS collaboration actively pursues covering more SUSY parameter space by employing new and ingenious analysis methods. Recent results show that inclusive searches are complemented by specialized searches for third generation sparticle production, boosted objects and VBF tagging. The new $\sqrt{s}=13 \mathrm{TeV}$ data to come will provide these methods, new and old alike, plenty of opportunities to be put to use.

\section{References}

[1] V. Khachatryan et al. [CMS Collaboration], Searches for supersymmetry using the $M_{T 2}$ variable in hadronic events produced in pp collisions at 8 TeV, JHEP 1505 (2015) 078 [arXiv:1502.04358 [hep-ex]].

[2] V. Khachatryan et al. [CMS Collaboration], Searches for third generation squark production in fully hadronic final states in proton-proton collisions at sqrt(s)=8 TeV, arXiv:1503.08037 [hep-ex].

[3] V. Khachatryan et al. [CMS Collaboration], Search for physics beyond the standard model in events with two leptons, jets, and missing transverse momentum in pp collisions at sqrt $(s)=8 \mathrm{TeV}$, JHEP 1504 (2015) 124 [arXiv:1502.06031 [hep-ex]].

[4] CMS Collaboration [CMS Collaboration], Search for electroweak SUSY with VBF tagging, CMS-PAS-SUS-14-005.

[5] https://twiki.cern.ch/twiki/bin/view/CMSPublic/PhysicsResultsSUS 